\title{
The ratio of small to large separations of acoustic oscillations as a diagnostic of the interior of solar-like stars
}

\author{
I. W. Roxburgh ${ }^{1,2}$ and S. V. Vorontsov ${ }^{1,3}$ \\ 1 Astronomy Unit, Queen Mary, University of London, Mile End Road, London E1 4NS, UK \\ 2 LESIA, Observatoire de Paris, place Jules Janssen, 92195 Meudon, France \\ 3 Institute of Physics of the Earth, B Gruzinskya 10, Moscow 123810, Russia
}

Received 7 May 2003 / Accepted 14 August 2003

\begin{abstract}
By considering stellar models with the same interior structure but different outer layers we demonstrate that the ratio of the small to large separations of acoustic oscillations in solar-like stars is essentially independent of the structure of the outer layers, and is determined solely by the interior structure. Defining the scaled Eulerian pressure perturbation $\psi_{\ell}(\omega, t)=$ $r p^{\prime} /(\rho c)^{1 / 2}$ we define the internal phase shift $\delta_{\ell}(\omega, t)$ through the relation $\omega \psi /(\mathrm{d} \psi / \mathrm{d} t)=\tan \left(\omega t-\pi \ell / 2+\delta_{\ell}\right)$. The $\delta_{\ell}$ are almost independent of acoustic radius $t=\int \mathrm{d} r / c$ outside the stellar core and can be determined as a continuous functions of $\omega$ from partial wave solutions for the interior - that is solutions of the oscillation equations for any $\omega$ that satisfy the Laplace boundary condition at a sufficiently large acoustic radius $t_{f}$ outside the stellar core. If the $\omega$ are eigenfrequencies then they satisfy the Eigenfrequency Equation $\omega T=(n+\ell / 2) \pi+\alpha(\omega)-\delta_{\ell}(\omega)$ where $\alpha(\omega)$ is the $\ell$ independent surface phase shift (Roxburgh \& Vorontsov 2000). Using this result we show that the ratio of small to large separations is determined to high accuracy solely by the internal phase shifts $\delta_{\ell}(\omega)$ and hence by the interior structure alone. The error in this result is estimated and shown to be smaller than that associated with the errors in the determination of the frequencies $(\approx 0.1-0.3 \mu \mathrm{Hz})$ from the upcoming space missions MOST, COROT and Eddington.
\end{abstract}

Key words. stars: oscillations

\section{Introduction}

The upcoming space missions MOST (Mathews 1998), COROT (Baglin et al. 1998) and Eddington (Roxburgh et al. 2000; Favata et al. 2000) will observe the photometric time series of flux integrated over the stellar disc, enabling us to determine the oscillation frequencies $v_{n \ell m}$ of modes of low degree $\ell$. The surface geometry of the modes is described by a spherical harmonic $Y_{\ell m}$, and $n$ is the radial order of the mode. For spherical stars the frequencies are $m$-degenerate $v_{n \ell m}=v_{n \ell}$, this degeneracy being lifted by rotation. Low degree modes penetrate deep inside the star, each frequency being a different functional of the variation of density, pressure and adiabatic exponent with radius. In principle, knowledge of a set of frequencies should provide knowledge on the internal structure that can be used to test and develop our understanding of stellar evolution. This is the goal of asteroseismology.

For acoustic p-modes in solar like stars combinations of frequencies often proposed for diagnostic purposes are the large separations $\Delta_{\ell}$, and small separations $d_{02}$ and $d_{13}$, defined by

$\Delta_{\ell}(n)=v_{n, \ell}-v_{n-1, \ell}$

$d_{02}(n)=\left(v_{n, 0}-v_{n-1,2}\right)$

Send offprint requests to: I. W. Roxburgh, e-mail: Roxburgh@qmul.ac.uk $d_{13}(n)=\left(v_{n, 1}-v_{n-1,3}\right)$

Additionally we will consider the separations $d_{01}$, initially defined as the 3 point separation $\left(v_{n, 1}-2 v_{n, 0}+v_{n+1,1}\right) / 2$ (Roxburgh 1993), but here defined as the smoother 5 point separations

$d_{01}(n)=\frac{1}{8}\left(v_{n-1,0}-4 v_{n-1,1}+6 v_{n, 0}-4 v_{n, 1}+v_{n+1,0}\right)$

$d_{10}(n)=-\frac{1}{8}\left(v_{n-1,1}-4 v_{n, 0}+6 v_{n, 1}-4 v_{n+1,0}+v_{n+1,1}\right)$.

The large separations for the standard solar reference modelS of Christensen-Dalsgaard et al. (1996) are shown in Fig. 1, whereas Fig. 2 shows the scaled small separations for the same model.

A plot of the average values of $d_{02}$ vs. $\Delta$ as a diagnostic tool was introduced by Christensen-Dalsgaard (1988) (see also Mazumdar \& Roxburgh 2003), and other combinations of frequencies were considered by Audard \& Provost (1994) and Audard et al. (1994).

In the asymptotic limit of high frequency Tassoul (1980) found that

$d_{\ell, \ell+2} \approx \frac{2 \ell+3}{4 \pi^{2} v_{n \ell} T}\left(\frac{c(R)}{R}-\int_{0}^{R} \frac{\mathrm{d} c}{r}\right) ; \quad T=\int_{0}^{R} \frac{\mathrm{d} r}{c}$ 


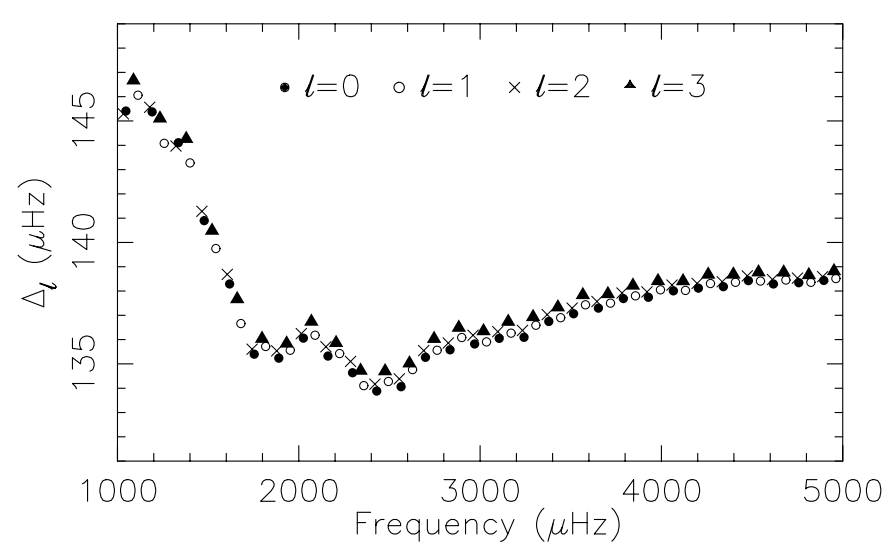

Fig. 1. Large separations $\Delta_{\ell}$ for ModelS and $\ell=0,1,2,3$.

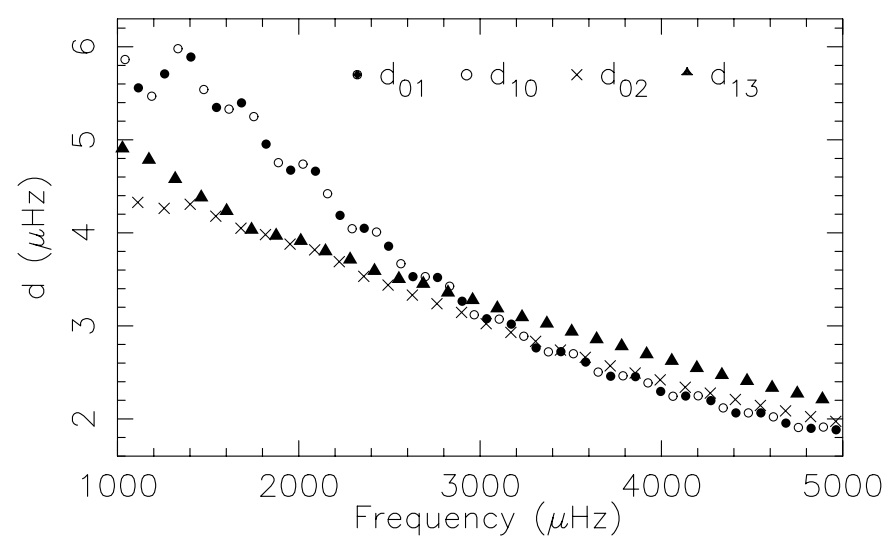

Fig. 2. Scaled small separations $d_{02} / 3, d_{13} / 5, d_{01}, d_{10}$ : ModelS.

being the acoustic radius of the star and $c$ the sound speed. The dependence on the derivative of $c$, which changes sign in the solar core, suggests that the small separations give a diagnostic of the deep interior of the star. In fact the Tassoul asymptotic result gives a poor fit both to the small separations of stellar models and to the observed values for the Sun. A much better fit was obtained by Roxburgh \& Vorontsov (1994) using a distorted wave Born approximation.

\section{Contribution of the outer layers of a star}

To examine the effect of the outer layers of a star on the separations we construct a set of 4 models with exactly the same interior structure but with different outer envelopes for $r \geq r_{f}$. That is $P(r), \rho(r), M_{r}(r)$ are unchanged for $r \leq r_{f}$. One model is modelS itself, the other three are:

Model A: $P, \rho, M_{r}$ unchanged for all $r$ but $\Gamma_{1}=5 / 3$ for all $r$. Since $\Gamma_{1} \approx 5 / 3$ for $r<0.95 R_{\odot}$ this is almost the same as just changing the value of $\Gamma_{1}$ for $r>0.95 R_{\odot}$.

Model B: For $r \geq r_{f}=0.9 R_{\odot}$ the structure of the envelope is determined by a linear variation of polytropic index $n=n_{0}+$ $n_{1}\left(r-r_{f}\right)$ with $n$ continuous at $r=r_{f}$. The model has a radius $1 R_{\odot}$ and mass of $1 M_{\odot}$ and $\Gamma_{1}$ is the same as in modelS.

Model C: For $r \geq r_{f}=0.72$ the envelope is adiabatic with $\Gamma_{1}=5 / 3$. This model has radius of $0.995 R_{\odot}$.

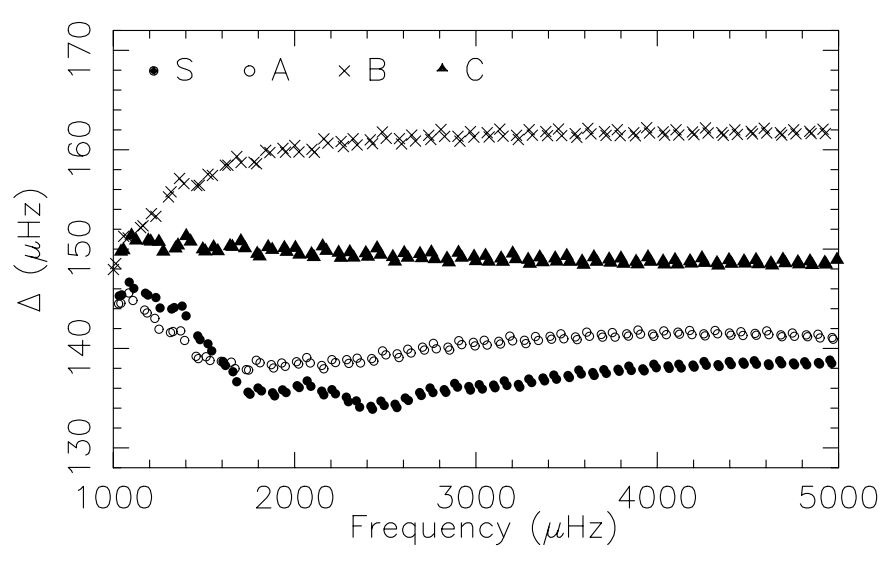

Fig. 3. Large separations $\Delta_{\ell}, \ell=0,1,2,3$, for all 4 models.

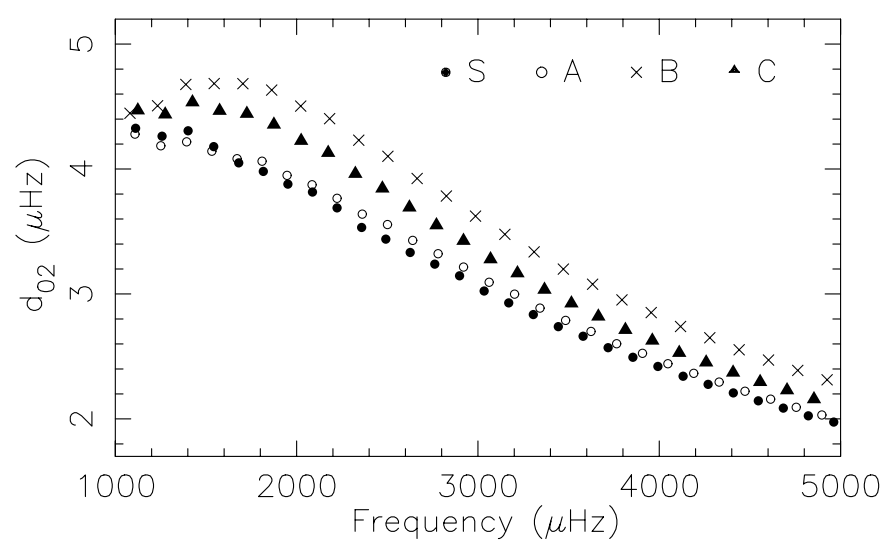

Fig. 4. Small separations $d_{02}$ for all 4 models.

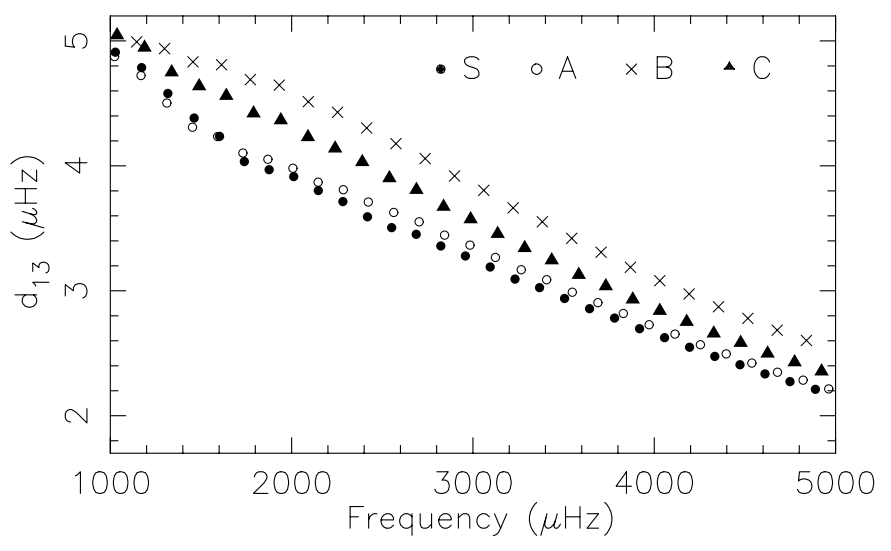

Fig. 5. Small separations $d_{13}$ for all 4 models.

Figure 3 shows the large separations $\Delta_{\ell}$ for all 4 models and for $\ell=0,1,2,3$. The small separations $d_{02}, d_{13}, d_{01}$ and $d_{10}$ are shown in Figs. 4-6. It is clear that the structure of the outer layers plays a significant role in determing both large and small separations.

\section{The ratio of small to large separations}

We define the ratios $r_{i j}$ of small to large separations as

$r_{02}(n)=\frac{d_{02}(n)}{\Delta_{1}(n)}, \quad r_{13}(n)=\frac{d_{13}(n)}{\Delta_{0}(n+1)}$ 


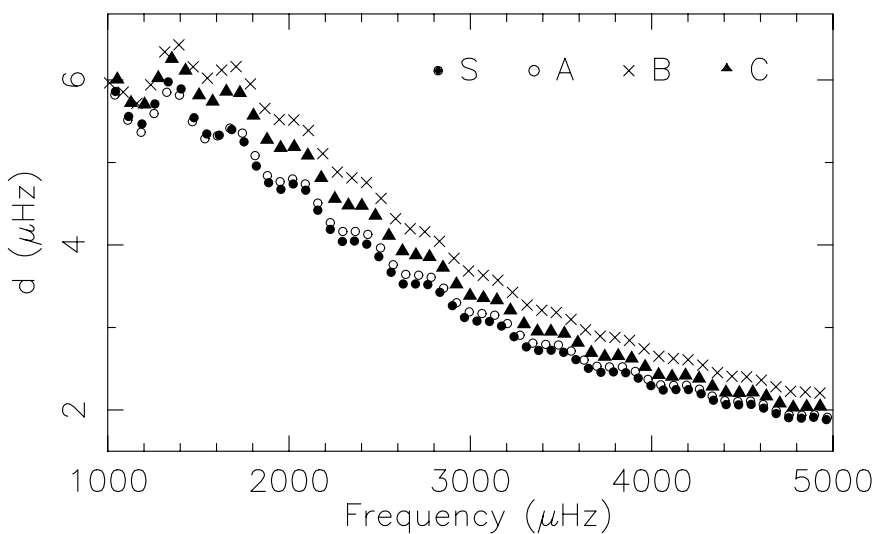

Fig. 6. Small separations $d_{01}, d_{10}$ for all 4 models.

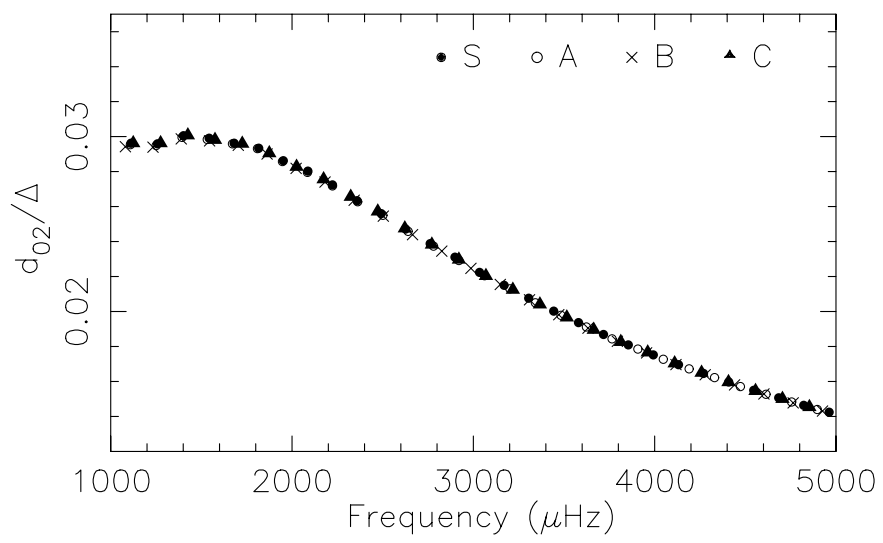

Fig. 7. Ratio $r_{02}=d_{02} / \Delta_{1}$ for all 4 models.

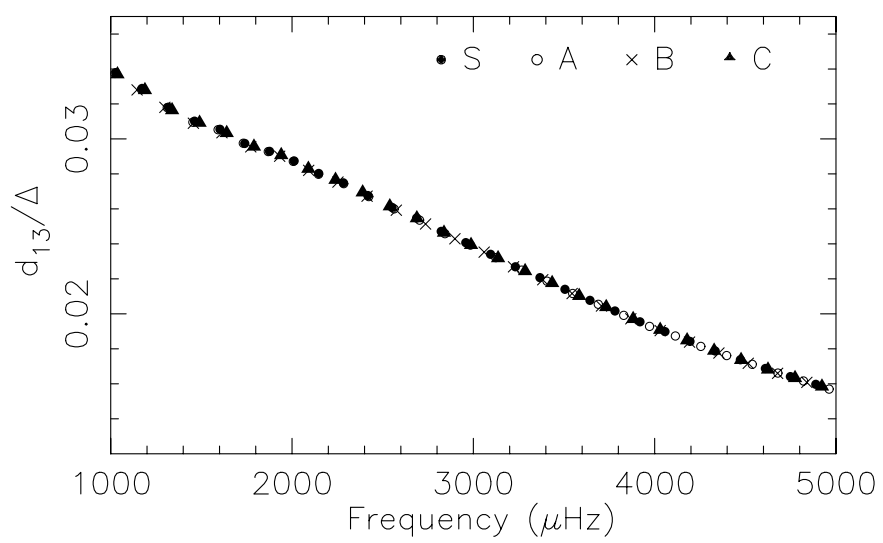

Fig. 8. Ratio $r_{13}=d_{13} / \Delta_{0}$ for all 4 models.

$r_{01}(n)=\frac{d_{01}(n)}{\Delta_{1}(n)} \quad r_{10}(n)=\frac{d_{10}(n)}{\Delta_{0}(n+1)}$

Figures 7-9 show these ratios for all 4 models. As can be seen from these figures the ratios $r_{i j}$ are essentially the same for all 4 models. Since the modified models are identical to the unmodified modelS in the inner layers, but differ in the outer layers, this demonstrates empirically that the ratios $r_{i j}$ of small to large separations are independent of the structure of the outer layers of a star, and therefore provide a diagnostic of the stellar interior alone.

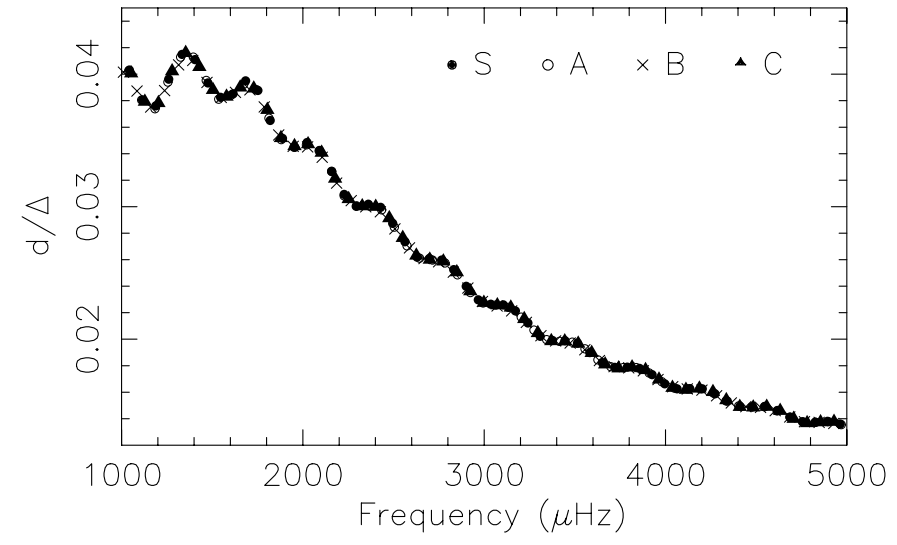

Fig. 9. Ratio $r_{01}=d_{01} / \Delta_{1}, r_{10}=d_{10} / \Delta_{0}$. for all 4 models.

\section{Phase shifts and the Eigenfrequency Equation}

To understand this result we introduce the concepts of phase shifts and partial waves, and derive the Eigen-frequency Equation of Roxburgh \& Vorontsov (2000).

The equations governing the oscillations of a spherical star can be expressed as (see e.g. Unno et al. 1979)

$\frac{\mathrm{d} \xi}{\mathrm{d} r}+\frac{2}{r} \xi-\frac{g}{c^{2}} \xi+\left(1-\frac{\ell(\ell+1) c^{2}}{\omega^{2} r^{2}}\right) \frac{p^{\prime}}{\rho c^{2}}=\frac{\ell(\ell+1)}{\omega^{2} r^{2}} \phi^{\prime}$

$\frac{\mathrm{d} p^{\prime}}{\mathrm{d} r}+\frac{g}{c^{2}} p^{\prime}+\left(N^{2}-\omega^{2}\right) \rho \xi+\rho \frac{\mathrm{d} \phi^{\prime}}{\mathrm{d} r}=0$

$\frac{\mathrm{d}^{2} \phi^{\prime}}{\mathrm{d} r^{2}}+\frac{2}{r} \frac{\mathrm{d} \phi^{\prime}}{\mathrm{d} r}-\frac{\ell(\ell+1)}{r^{2}} \phi^{\prime}=4 \pi G \rho\left(\frac{p^{\prime}}{\rho c^{2}}+\frac{N^{2}}{g} \xi\right)$

where the perturbations in radius $\delta r$, pressure $\delta P$ and gravitational potential $\delta \Phi$ are decomposed in the form

$\delta r=\xi(r) Y_{\ell m} \mathrm{e}^{\mathrm{i} \omega t}, \delta P=p^{\prime}(r) Y_{\ell m} \mathrm{e}^{\mathrm{i} \omega t}, \delta \Phi^{\prime}=\phi(r) Y_{\ell m} \mathrm{e}^{\mathrm{i} \omega t}$

with $Y_{\ell m}$ spherical harmonics and $\omega$ the angular frequency $(\omega=2 \pi \nu)$. The sound speed $c$, Brunt-Väisälä frequency $N$, and acceleration due to gravity $g$, are defined as

$c^{2}=\Gamma_{1} \frac{P}{\rho}, \quad N^{2}=\frac{g^{2}}{c^{2}}\left(1-\Gamma_{1} \frac{\mathrm{d} \log \rho}{\mathrm{d} \log P}\right) g=\frac{G M_{r}}{r^{2}}$.

These equations are governed by boundary conditions of regularity at the centre, and that at the surface the potential $\phi^{\prime}$ matches onto the corresponding $\ell$ dependent solution of Laplace's equation, and the wave is reflected high in the atmospheric layers. This reflective wave condition is often approximated to the vanishing of the Lagrangian pressure perturbation $p^{\prime}-\rho g \xi=0$ at $r=R$ (see e.g. Unno et al. 1979; Vorontsov \& Zharkov 1989).

We now define the scaled pressure perturbation $\psi$, and acoustic radius $t$, as

$\psi=\frac{p^{\prime} r}{(\rho c)^{1 / 2}}, \quad t=\int_{0}^{r} \frac{\mathrm{d} r}{c}$

The solution $\psi_{\ell}(t)$ for a particular eigenmode is shown in Fig. 10. $\psi_{\ell}(t)$ behaves like a Spherical Bessel function $J_{\ell}(\omega t)$ in the interior which in turn behaves like $\sin (\omega t-\pi \ell / 2)$ for 


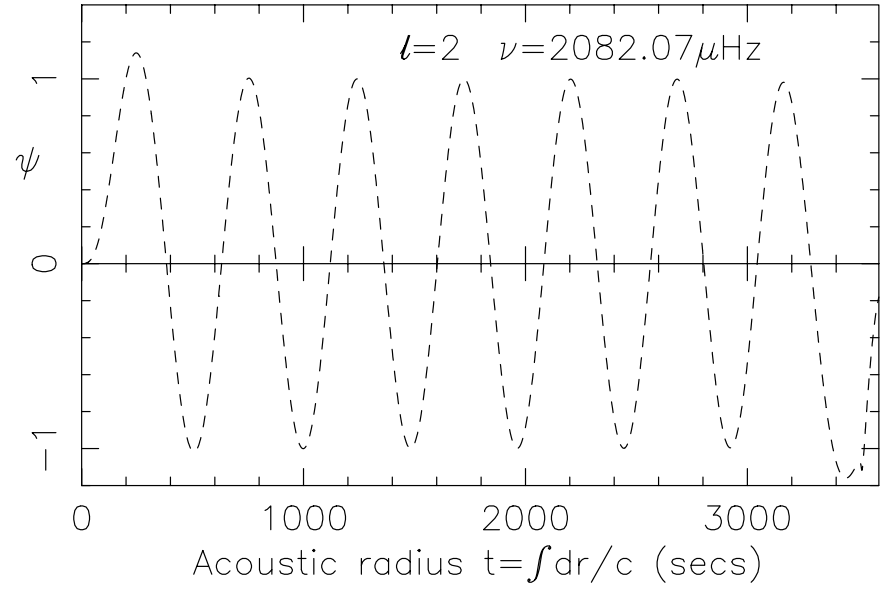

Fig. 10. Eigensolution $\psi_{\ell}(t) ; \ell=2, v=2082.07 \mu \mathrm{Hz}$.

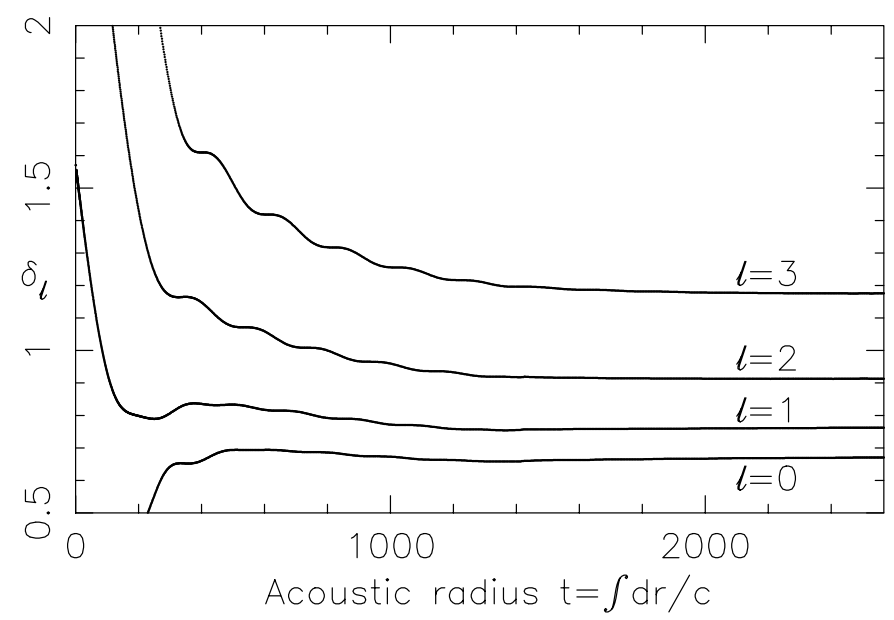

Fig. 11. Inner phase shifts $\delta_{\ell}$ versus acoustic radius at $v=2500 \mu \mathrm{Hz}$.

$\omega t>1$. The $J_{\ell}$ are the exact solutions for a uniform non gravitating sphere (Rayleigh 1896) and the p-mode eigenfunctions for a real star can be represented as distorted Bessel functions (Roxburgh \& Vorontsov 1994).

We now represent the $\psi$ in terms of internal phase shifts $\delta_{\ell}(\omega, t)$ defined by

$\chi_{\ell}(\omega, t)=\frac{\omega \psi}{\mathrm{d} \psi / \mathrm{d} t}=\tan \left[\omega t-\ell \pi / 2+\delta_{\ell}(\omega, t)\right]$

where $\omega=2 \pi v$ is the angular frequency. The introduction of the term $\ell \pi / 2$ is for convenience and captures the fact that the eigenfunctions behave like Spherical Bessel functions $J_{\ell}(\omega t)$ in the interior.

In Fig. 11 we show the variation of the $\delta_{\ell}(\omega, t)$ with acoustic radius $t$ for modelS; the $\delta_{\ell}$ become almost independent of $t$ outside the stellar core. Figure 12 shows the variation of $\delta_{\ell}(\omega)$ at $r / R_{\odot}=0.9$.

The $\delta_{\ell}$ can be defined as continuous functions of $\omega$ using partial wave solutions of the equations governing the oscillations, that is solutions for any $\omega$ out to some $t=t_{f}$ that satisfy the central conditions of regularity, and where the perturbation in gravitational potential $\phi^{\prime}$ matches on to the appropriate $\ell$ dependent solution of Laplace's equation at $t_{f}$. In the outer layers of a star the density $\rho$ is very small and the partial wave

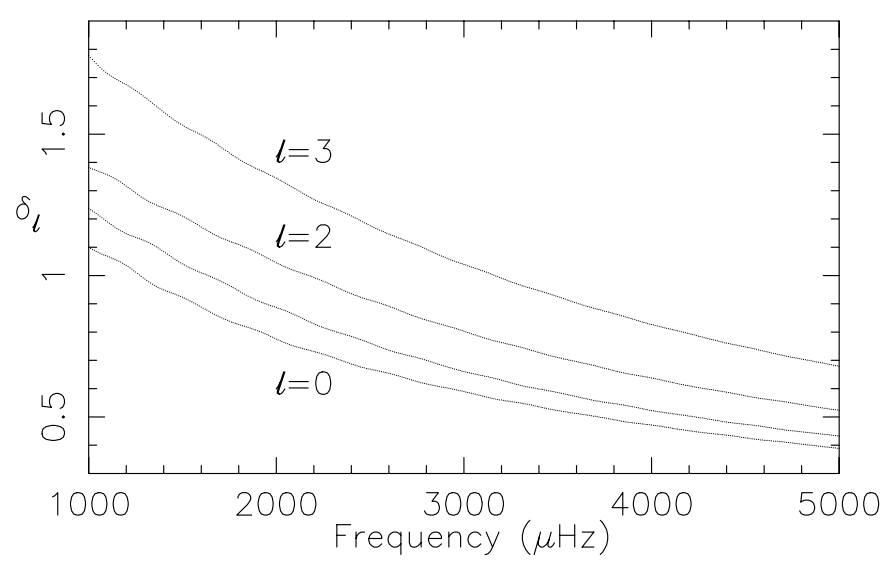

Fig. 12. Inner phase shifts $\delta_{\ell}(v)$ at $r / R_{\odot}=0.9$.

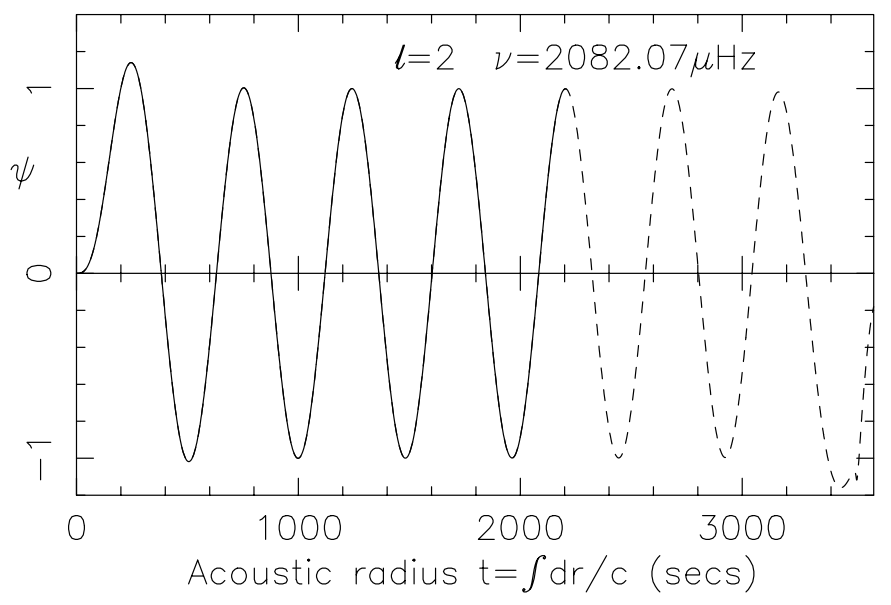

Fig. 13. Partial wave solution superposed on eigensolution.

solutions reproduce the actual eigensolutions to very high accuracy. This is seen in Fig. 13 where we have superposed the partial wave solution out to $r=0.9 R_{\odot}$ on the full eigensolution.

We similarly define surface phase shifts $\alpha_{\ell}$ by

$\chi_{\ell}(\omega, t)=\frac{\omega \psi}{\mathrm{d} \psi / \mathrm{d} t}=-\tan \left[\omega t_{e}-\alpha_{\ell}\left(\omega, t_{e}\right)\right]$

where $t_{e}=T-t$ is the acoustic depth. In Fig. 14 we show the surface phase shifts $\alpha_{\ell}$ as a function of acoustic depth $t_{e}$, and in Fig. 15 the variation with frequency at a depth of $r / R_{\odot}=0.9$; the $\alpha_{\ell}$ collapse to a single function of frequency which is almost independent of acoustic depth $t_{e}$ below the near surface layers. This behaviour in the outer layers is well known (e.g. Gough 1986; Vorontsov \& Zarkhov 1989; Christensen-Dalsgaard \& Perez Hernandez 1992) and is due to the very weak dependence of the eigensolution on $\ell$ when $\ell(\ell+1) c^{2} /(\omega r)^{2} \ll 1$ for small $c$ and large $r$, and the perturbation in gravitational potential is also small.

Equations (15) and (16) are two representations of the same function $\chi_{\ell}(\omega, t)$; equating these at some intermediate acoustic radius $t$, and recalling that $\tan (\theta+n \pi)=\tan (\theta)$, we obtain the Eigenfrequency Equation

$\omega_{n, \ell} T=(n+\ell / 2) \pi+\alpha\left(\omega_{n, \ell}\right)-\delta_{\ell}\left(\omega_{n, \ell}\right)$ 


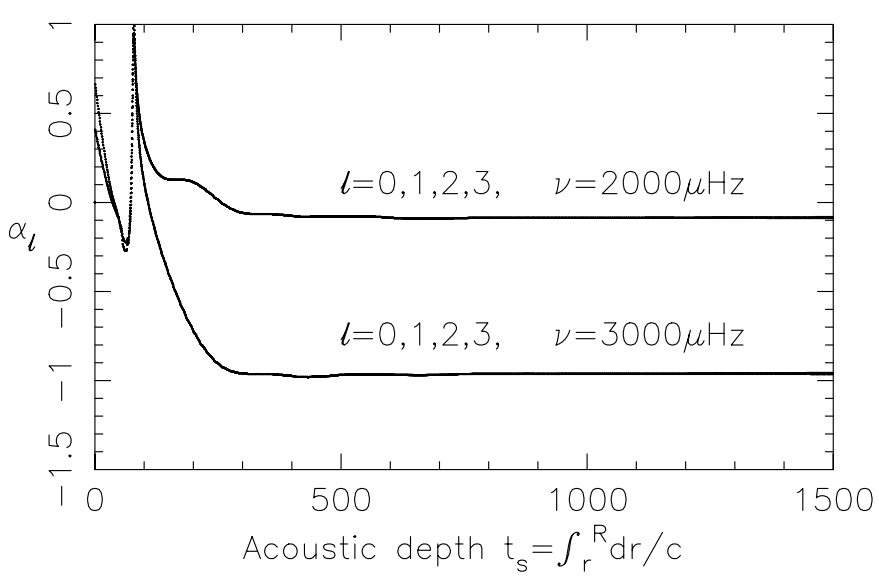

Fig. 14. Outer phase shifts $\alpha_{\ell}$ as a function of acoustic depth for frequencies $v=2000,3000 \mu \mathrm{Hz}$ and $\ell=0,1,2,3$.

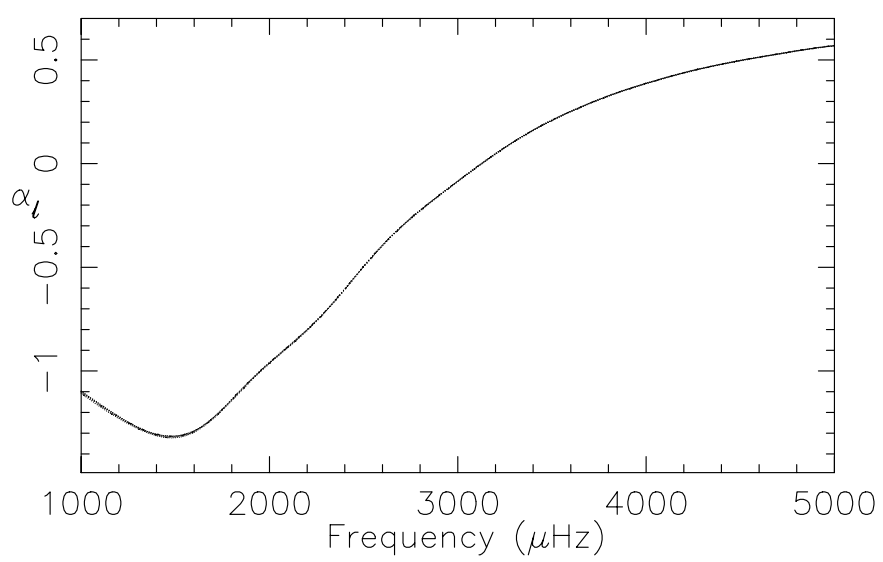

Fig. 15. Outer phase shifts $\alpha_{\ell}$ as a function of frequency at $r / R_{\odot}=0.9$ for $\ell=0,1,2,3$.

where $T$ is the acoustic radius, $\delta_{\ell}(\omega)$ the internal phase shifts, $\alpha(\omega)$ the surface phase shift, and $n$ an integer.

\section{Separations in terms of phase shifts}

We now derive expressions for the large and small separations in terms of the phase shifts $\alpha(\omega), \delta_{\ell}(\omega)$, using the Eigenfrequency Equation (17).

To lowest order we neglect the variation of $\alpha$ and $\delta_{\ell}$ with $\omega$ between neighbouring frequencies and obtain the approximations

$\Delta_{\ell}=\frac{1}{2 \pi}\left[\omega_{n, \ell}-\omega_{n-1, \ell}\right]=\frac{1}{2 T}$

$\frac{d_{02}}{\Delta_{1}}=\frac{1}{\pi}\left(\delta_{2}-\delta_{0}\right), \quad \frac{d_{13}}{\Delta_{0}}=\frac{1}{\pi}\left(\delta_{3}-\delta_{1}\right)$

$\frac{d_{01}}{\Delta_{1}}=\frac{1}{\pi}\left(\delta_{1}-\delta_{0}\right), \quad \frac{d_{10}}{\Delta_{0}}=\frac{1}{\pi}\left(\delta_{1}-\delta_{0}\right)$.

The ratios depend only on the internal phase shifts $\delta_{\ell}$ and hence only on the interior structure of the star.
For the calculation to higher order we take, as an example, the ratio $r_{02}$. We first define a mean angular frequency $\omega$ and angular large separation $\Delta$ such that

$\omega_{n, 1}=\omega+\frac{1}{2} \Delta, \quad \omega_{n-1,1}=\omega-\frac{1}{2} \Delta$

and expand $\alpha$ and $\delta_{\ell}$ around $\omega$ to obtain

$\left(T-\frac{\partial \alpha}{\partial \omega}+\frac{\partial \delta_{1}}{\partial \omega}\right) \Delta=\pi+\frac{\partial^{3}}{\partial \omega^{3}}\left(\alpha-\delta_{1}\right) \frac{\Delta^{3}}{24}+\ldots$

We now define the small separations in angular frequency $e, d$, as

$\omega_{n, 0}-\omega=e, \omega_{n, 0}-\omega_{n-1,2}=d$.

On expanding $\alpha$ and $\delta_{\ell}$ about $\omega$ we obtain

$T d=\left[\delta_{2}-\delta_{0}\right]+\frac{\partial \alpha}{\partial \omega} d+\frac{\partial \delta_{2}}{\partial \omega}(e-d)-\frac{\partial \delta_{0}}{\partial \omega} e+O\left(d^{2}\right)$

which, on neglecting terms of order $d^{2}$, gives

$\left(T-\frac{\partial \alpha}{\partial \omega}+\frac{\partial \delta_{1}}{\partial \omega}\right) d=\left(\delta_{2}-\delta_{0}\right)+\left(\frac{\partial \delta_{1}}{\partial \omega}-\frac{\partial \delta_{0}}{\partial \omega}\right) d$

where $\delta_{2}-\delta_{0}$ is evaluated at $\omega_{n-1,2}=\omega+e-d$ and all other terms are evaluated at $\omega$.

Dividing Eq. (25) by Eq. (22) gives

$\frac{d_{02}}{\Delta_{1}}=\frac{d}{\Delta}=\frac{1}{\pi}\left(\delta_{2}-\delta_{0}\right)(1+\epsilon)$

where, on using the zero order result $\pi d=\Delta\left(\delta_{2}-\delta_{0}\right.$ ) (cf. Eq. (19)), the error term can be expressed as

$\epsilon=\frac{1}{\pi}\left(\frac{\partial\left(\delta_{1}-\delta_{0}\right)}{\partial \omega} \Delta-\frac{\partial^{3}}{\partial \omega^{3}}\left(\alpha-\delta_{1}\right) \frac{\Delta^{3}}{24}\right)$

or, on converting to $v$,

$\epsilon=\frac{1}{\pi}\left(\frac{\partial\left(\delta_{1}-\delta_{0}\right)}{\partial v} \Delta_{1}-\frac{\partial^{3}}{\partial v^{3}}\left(\alpha-\delta_{1}\right) \frac{\Delta_{1}^{3}}{24}\right)$.

Referring to Figs. 12 and 15 with $\Delta_{1} \approx 140 \mu \mathrm{Hz}$ we can estimate the value of $\epsilon \approx 5 \times 10^{-3}$ at low frequencies, decreasing at higher frequencies over the frequency range $1000-5000 \mu \mathrm{Hz}$. To this order of accuracy the ratio of small to large separation is given by

$\frac{d_{02}(n)}{\Delta_{1}(n)}=\frac{1}{\pi}\left[\delta_{2}(v)-\delta_{0}(v)\right]$

the same as obtained by the lower order analysis (Eq. (19)), and is determined solely by the interior structure of the star, independent of the structure of the outer layers.

A parallel analysis can be carried through for the other ratios of small to large separations with the same conclusions; to high accuracy the ratios depend only on the inner phase shifts and hence on the interior structure of the star.

The upcoming space missions MOST, COROT and Eddington have as one of their goals the determination of frequencies of solar like oscillations to a precision of $0.1-0.3 \mu \mathrm{Hz}$. Since the small separations $d_{02}$ are of order $10 \mu \mathrm{Hz}$ the determinations of the ratio $d_{02} / \Delta_{1}$ have an error of order $1.5 \%$ which is somewhat greater than the error $\epsilon$ in the above analysis. 


\section{Conclusions}

The oscillation frequencies, large, and small separations depend on the structure of both the inner and outer layers of a star, so model fitting and testing techniques to probe the interior structure of stars are dependent on our having a good understanding of the structure of the outer layers. But these are just the layers where our ignorance is greatest; non adiabatic convection is important but not understood, the oscillations are non adiabatic in the surface layers, and the structure of real stellar atmospheres is poorly understood. For example the oscillation frequencies predicted by the reference solar modelS differ from the observed values by up to $10 \mu \mathrm{Hz}$ at the higher end of the observed frequency range.

By constructing stellar models with the same interior structure $P(r), \rho(r), M_{r}(r)$ but with different outer layers we found that although the small and large separations differ between the models, the ratios of small to large separations are very closely equal for all the models. We then showed that the eigenmodes of oscillation can be expressed in terms of inner phase shifts $\delta_{\ell}(v)$ and an outer $\ell$ independent phase shift $\alpha(v)$ which satisfy an Eigenfrequency Equation

$2 \pi v_{n, \ell} T=(n+\ell / 2) \pi+\alpha\left(v_{n, \ell}\right)-\delta_{\ell}\left(v_{n, \ell}\right)$.

Using this equation we are able to show that to an accuracy $\approx 5 \times 10^{-3}$ the ratios of small to large separations depend only on the inner phase shifts $\delta_{\ell}(v)$ which are determined solely by the interior structure of the star, and are uninfluenced by the unknown structure of the outer layers. Hence these ratios give valuable diagnostics that can be used to test models of the interior structure of stars using the high precision data sets of p-mode oscillation frequencies from the upcoming space missions MOST, COROT and Eddington.

The above analysis can be improved by least squares fitting an observed frequency set to Eq. (30) to extract the inner phase shift differences $\delta_{\ell}(v)-\delta_{0}(v)$ and the combination $2 \pi v T-\alpha(v)+\delta_{0}(v)$. These functions can then be used as the basis of an inversion technique to map the interior structure of a star even though we cannot map the outer layers (Roxburgh \& Vorontsov 2002a), and to reveal signatures of the location of regions of rapid change in acoustic variables such as the HeII ionisation zone and the base of a convective envelope (Roxburgh \& Vorontsov 2001; Roxburgh 2002).

The property that the phases of the inner partial waves $\chi_{\ell}(\omega, t)$, defined in Eq. (15), collapse onto a function independent of $\ell$ in the outer layers, is the basis of the Differential Response Inversion Technique which has yielded high resolution inversion of helioseismic data (Vorontsov 1998, 2001), and has been successfully applied to simulated small frequency sets for solar-like stars (Roxburgh \& Vorontsov 2002b,c). These techniques are currently being further refined and will be reported in subsequent publications.
Acknowledgements. This work has been supported in part by the UK Particle Physics and Astronomy Research Council under grants PPA/G/O/1999/00576 and PPA/G/O/2001/00486.

\section{References}

Audard, N., \& Provost, J. 1994, A\&A, 282, 73

Audard, N., Provost, J. \& Christensen-Dalsgaard, J. 1994, A\&A, 297, 427

Baglin, A., \& the COROT Team, New Eyes to see inside the Sun and Stars, IAU Symp., 185, 301

Christensen-Dalsgaard, J. 1988, in Advances in Helio and Asteroseismology, ed. J. Christensen-Dalsgaard, \& S. Fransden (Reidel), 295

Christensen-Dalsgaard, J., \& Perez Hernandez, F. 1992, MNRAS, 257, 62

Christensen-Dalsgaard, J., Däppen, W., Ajukov, S. V. et al. 1996, Science, 272, 1296

Favata, F., Roxburgh, I. W., \& Christensen-Dalsgaard, J. (ed.) 2000, Eddington, ESA Assessment Study Report: A Mission to Map Stellar Evolution through Oscillations and to Find Habitable Planets, ESA-SCI(2000)8

Gough, D. O. 1986, in Seismology of the Sun and the Distant Stars, ed. Gough, D. O. (Dordrecht: Reidel), 125

Mathews, J. M. 1998, in Structure and Dynamics of the Interior of the Sun and Sun-like stars, SOHO6/GONG98 Workshop, ESA SP-418, 395

Mazumdar, A., \& Roxburgh, I. W. 2003, Asteroseismology Across the H-R Diagram, ed. M. Thompson, M. Cunha, \& M. Monteiro (Kluwer Academic Publishers), 477

Rayleigh, Lord (J. W. Strutt) 1896, The Theory of Sound (Cambridge University Press)

Roxburgh. I. W. 1993, in PRISMA, Report of Phase A Study, ed. T. Appourchaux, et al., ESA SCI(93), 31

Roxburgh, I. W., Christensen-Dalsgaard, J., \& Favata, F. (ed.) 2000, Eddington - A Stellar Physics and Planet Finder Explorer. F2/F3 Proposal submitted to ESA

Roxburgh, I. W. 2002, Proceedings of the First Eddington Workshop, ed. F. Favata, I. W. Roxburgh, \& D. Galadi, ESA SP-485, 75

Roxburgh, I. W., \& Vorontsov, S. V. 1994, MNRAS, 267, 297

Roxburgh, I. W., \& Vorontsov, S. V. 2000, MNRAS, 317, 141

Roxburgh, I. W., \& Vorontsov, S. V. 2001, MNRAS, 322, 85

Roxburgh, I. W., \& Vorontsov, S. V. 2002a, ESA SP-485, 341

Roxburgh, I. W., \& Vorontsov, S. V. 2002b, ESA SP-485, 349

Roxburgh, I. W., \& Vorontsov, S. V. 2002c, ESA SP-485, 337

Tassoul. M. 1980, ApJS, 43, 469

Unno, W., Osaki, Y., Ando, H., et al. 1979, Nonradial Oscillations of Stars (Tokyo: Univ. of Tokyo Press)

Vorontsov, S. V. 1998, in Sounding Solar and Stellar Interiors, ed. J., Provost, \& F.-X. Schmider (Nice), 135

Vorontsov, S. V. 2001, in Helio- and Asteroseismology at the dawn of the Millenium, ed. A. Wilson, ESA SP-464, 563

Vorontsov, S. V., \& Zarkhov, V. 1989, Soviet Sci. Rev. E, Astrophys. Spa. Sci. Rev., 7, 1 